\title{
FOXD1 is targeted by miR-30a-5p and miR-200a-5p and suppresses the proliferation of human ovarian carcinoma cells by promoting p21 expression in a p53-independent manner
}

\author{
YU WANG $^{1 *}$, CHUNPING QIU $^{1 *}$, NAN LU $^{2}$, ZHAOJIAN LIU $^{3}$, CHENGJUAN JIN $^{1}$, CHENGGONG SUN $^{1}$, \\ HUALEI BU ${ }^{1}$, HONGFENG YU ${ }^{4}$, SAMINA DONGOL ${ }^{1}$ and BEIHUA KONG ${ }^{1}$ \\ ${ }^{1}$ Department of Obstetrics and Gynecology, Qilu Hospital of Shandong University, Jinan, Shandong 250012; \\ ${ }^{2}$ Institute of Diagnostics and ${ }^{3}$ Department of Cell Biology, School of Medicine, \\ Shandong University, Jinan, Shandong 250012; ${ }^{4}$ Department of Obstetrics and Gynecology, \\ Zhenjiang First People's Hospital, Zhenjiang, Jiangsu 212002, P.R. China
}

Received December 21, 2017; Accepted March 19, 2018

DOI: 10.3892/ijo.2018.4359

\begin{abstract}
High-grade serous ovarian carcinoma (HGSOC) accounts for the highest number of deaths among patients with epithelial ovarian cancer. However, the molecular mechanisms underlying HGSOC tumorigenesis are currently unclear. In the present study, a lentiviral expression system was employed to manipulate forkhead box D1 (FOXD1) expression in ovarian cancer cells. Immunohistochemical staining was used to examine the expression of FOXD1 in tissue samples. Clonogenic and MTT assays were employed to evaluate cell proliferation, and flow cytometry was applied for cell cycle analysis. Dual-luciferase reporter and chromatin immunoprecipitation assays were used to determine the role of FOXD1 in regulating p21 expression. The results demonstrated that FOXD1 expression was downregulated in HGSOC, and high expression levels of FOXD1 were found to be a predictor of good prognosis. FOXD1 significantly inhibited the proliferation of human ovarian cancer cells and induced cell cycle arrest at G1 phase in vitro. In addition, exogenous FOXD1 expression inhibited ovarian cancer cell growth in vivo. Furthermore, microRNA (miR)-30a-5p and miR-200a-5p were observed to be upregulated in HGSOC, and function as direct negative regulators of FOXD1 by targeting its 3'-untranslated region. The present study also revealed that FOXD1 promotes p21 expression in a p53-independent manner. In conclusion, the results of the present study indicate a direct association
\end{abstract}

Correspondence to: Professor Beihua Kong, Department of Obstetrics and Gynecology, Qilu Hospital of Shandong University, 107 Wenhua Xi Road, Jinan, Shandong 250012, P.R. China

E-mail: kongbeihua@sdu.edu.cn

*Contributed equally

Key words: high-grade serous ovarian carcinoma, forkhead box D1, proliferation, p21, microRNA-30a-5p, microRNA-200a-5p, prognosis between FOXD1 and p21 that may be mediated by miR-30a-5p and miR-200a-5p. The authors hypothesize that FOXD1 may serve as a biomarker or therapeutic target in HGSOC.

\section{Introduction}

Ovarian cancer is considered to be the most lethal gynecologic tumor. In China, $\sim 52,100$ new cases of ovarian cancer were diagnosed and 22,500 mortalities occurred due to ovarian cancer in 2015 (1). Compared with China, the incidence and mortality rates in Europe and North America are higher (2). Epithelial ovarian cancer is divided into the following five major pathological subtypes: High-grade serous $(70 \%)$, low-grade serous $(<5 \%)$, endometrioid $(10 \%)$, clear cell $(10 \%)$ and mucinous $(3 \%)(3,4)$. High-grade serous ovarian carcinoma (HGSOC) accounts for 70-80\% of ovarian cancer-associated mortalities (5). Therefore, gaining an improved understanding of the molecular mechanisms underlying HGSOC is critical.

Forkhead box D1 (FOXD1), also known as FREAC-4, belongs to the forkhead box family and functions as a transcription factor (6). According to previous reports $(7,8)$, it serves an important role in the formation of the retina and nephrons during embryogenesis. In addition, downregulation of FOXD1 decreases the reprogramming efficiency and inhibits the expression of reprogramming-associated genes in mouse embryonic fibroblasts (9). FOXD1 also serves a role in the development of different cancers. In lung, breast and brain cancers, FOXD1 functions as an oncogene and promotes cell proliferation (10-12). In hepatocellular carcinoma, microarray analysis has demonstrated that FOXD1 is one of ten transcription factors involved in tumorigenesis (13). By contrast, FOXD1 may function as a tumor suppressor in ovarian cancer. Jiang et al (14) analyzed three GSE cohorts (GSE14001, GSE15578 and GSE12172) and demonstrated that FOXD1 is downregulated in ovarian cancer. The same study identified FOXD1 as one of the top 20 differentially expressed genes between abnormal and normal ovarian epithelial tissues (14).

p21 (also known as WAF1/CIP1 and cyclin-dependent kinase inhibitor 1A) is a general G1 phase cell cycle inhibitor, 
which is regulated by $\mathrm{p} 53$-dependent or p53-independent signaling pathways (15). The function of p21 as an inhibitor of the cell cycle via p53 was a landmark discovery in molecular biology in the early 1990 s $(16,17)$. Although p 21 is widely known to be a crucial effector of p53 and an inhibitor of the cell cycle, it is now evident that $\mathrm{p} 21$ is regulated by a number of additional signaling pathways $(18,19)$. p21 mutations are rare in human cancer $(20,21)$. Increased expression of p21 has been associated with a favorable outcome in many cancer types (22-25).

MicroRNAs (miRNAs/miRs) are endogenous short ( 22 nucleotide) single-stranded RNAs that regulate the translation of target genes by promoting mRNA decay. miRNAs recognize target mRNAs by base-pairing with their complementary seed sequences (typically nucleotides 2-7) in the 3'-untranslated region (UTR) (26). miRNAs function as tumor suppressors or oncogenes during carcinogenesis and tumor development. Specifically, the miR-30 and miR-200 families are known to function as tumor suppressors in a number of cancer types by inhibiting tumor cell proliferation and metastasis (27-29); however, several studies have demonstrated that these miR families may serve an oncogenic role in ovarian cancer (30-36).

In the present study, the expression of FOXD1 was analyzed in patients with HGSOC, and its role in the proliferation of ovarian cancer cells was investigated using in vitro and in vivo studies. The results indicated that FOXD1 was downregulated in HGSOC, and it suppressed ovarian cancer cell proliferation via targeting $\mathrm{p} 21$. In addition, the expression of miR-30a-5p and miR-200a-5p was elevated in patients with HGSOC, and FOXD1 was demonstrated to be a direct target of these miRNA sequences.

\section{Materials and methods}

Patients and tissue samples. A total of 140 HGSOC tissue specimens were collected for the purposes of this study. The tissue microarray (TMA) included 120 HGSOC samples and these tissues were collected from female patients admitted to Qilu Hospital (Jinan, China) from May 2006 to July 2013. The HGSOC samples $(n=20)$ used for western blot and reverse transcription-quantitative polymerase chain reaction (RT-qPCR) analyses were collected from patients admitted to Qilu hospital from September 2014 to July 2015. All HGSOC specimens were collected from patients with primary ovarian cancer that had not received chemotherapy prior to surgery. The fresh-frozen normal fallopian tube (FT, $n=11)$ tissues were obtained from female patients with benign gynecological tumors that had received a hysterectomy with bilateral salpingo-oophorectomy at Qilu hospital from September 2014 to July 2015. The tumor diagnoses were verified by two gynecological pathologists. Ethical approval was obtained from Ethics Committee of Shandong University Qilu Hospital (Jinan, China). All patients provided written informed consent.

Cell lines and culture conditions. 293T cells, and the human ovarian cancer cell lines, A2780 and HO8910, were purchased from the China Center for Type Culture Collection (Wuhan, China). Human ovarian cancer cell lines, SKOV3, OVCAR3, CAOV3 and the p53-null H1299 human lung cancer cell line, were purchased from the American Type Culture Collection (Manassas, VA, USA). A2780, HO8910 and H1299 cells were cultured in RPMI-1640 supplemented with $10 \%$ fetal bovine serum (FBS; both purchased from Gibco; Thermo Fisher Scientific, Inc., Waltham, MA, USA); OVCAR3 cells were maintained in RPMI-1640 supplemented with $20 \% \mathrm{FBS}$ and $0.01 \mathrm{mg} / \mathrm{ml}$ bovine insulin (Sigma-Aldrich; Merck KGaA, Darmstadt, Germany); SKOV3 cells were cultured in McCoy's 5A medium (Thermo Fisher Scientific, Inc.) supplemented with $10 \%$ FBS; CAOV3 cells were cultured in Dulbecco's modified Eagle's medium (Gibco; Thermo Fisher Scientific, Inc.) supplemented with 10\% FBS. In addition, $100 \mathrm{U} / \mathrm{ml}$ penicillin and $100 \mu \mathrm{g} / \mathrm{ml}$ streptomycin (Sigma-Aldrich; Merck KGaA) were added to the complete culture medium for all cell lines. All cells were cultured at $37^{\circ} \mathrm{C}$ with $5 \% \mathrm{CO}_{2}$ in a humidified incubator.

RNA extraction and RT- $q P C R$ analysis. Total RNA was extracted from fresh-frozen HGSOC and FT tissue samples, as well as OVCAR3 cells transfected with $\mathrm{miR}-30 \mathrm{a}-5 \mathrm{p} / \mathrm{miR}-200 \mathrm{a}-5 \mathrm{p}$ mimics or inhibitors, using TRIzol reagent according to manufacturer's instructions (Invitrogen; Thermo Fisher Scientific, Inc.). Total RNA (1,000 ng) was reverse transcribed to cDNA using the PrimeScript RT reagent kit or the Mir-X miRNA FirstStrand Synthesis kit (both purchased from Takara Biotechnology, Co., Ltd., Dalian, China) according to manufacturer's instructions. qPCR analysis was performed using the SYBR Premix Ex Taq (Takara Biotechnology, Co., Ltd.). The qPCR reaction was performed using the StepOnePlus ${ }^{\mathrm{TM}}$ Real-Time PCR System (Thermo Fisher Scientific, Inc.). The thermal cycling parameters were as follows: $30 \mathrm{sec}$ at $95^{\circ} \mathrm{C}$ followed by 43 cycles at $95^{\circ} \mathrm{C}$ for $5 \mathrm{sec}$ and $60^{\circ} \mathrm{C}$ for $30 \mathrm{sec}$. The primer sequences employed were as follows: FOXD1, forward, 5'-GATCTGTGAGTTCATCAGC GGC-3' and reverse, 5'-TGACGAAGCAGTCGTTGAGCGA-3'; $\beta$-actin, forward, 5'-CATGTACGTTGCTATCCAGGC-3' and reverse, 5'-CTCCTTAATGTCACGCACGAT-3'; miR-30a-5p, forward, 5'-TGTAAACATCCTCGACTGGAAG-3' and reverse, mRQ 3' Primer (provided in Mir-X miRNA First-Strand Synthesis kit; Takara Biotechnology, Co., Ltd.); miR-200a-5p, forward, 5'-CATCTTACCGGACAGTGCTGGA-3' and reverse, mRQ 3' Primer; U6, forward, 5'-GCTTCGGCAGCACATA TACTAAAAT-3' and reverse, mRQ 3' Primer. The expression of $\beta$-actin and U6 was used as the internal control. Data analysis was performed using the $2^{-\Delta \Delta C q}$ method (37).

Western blotting. Protein from fresh-frozen HGSOC and FT tissue samples was isolated using TRIzol reagent according to manufacturer's instructions (Invitrogen; Thermo Fisher Scientific, Inc.). The HGSOC and FT tissue samples were divided into smaller sections $(\sim 0.1 \mathrm{~g})$ and lysed with TRIzol for $10 \mathrm{~min}$ at room temperature. The lysate was centrifuged at $12,000 \mathrm{x} \mathrm{g}$ for $10 \mathrm{~min}$ at $4^{\circ} \mathrm{C}$ and the phenol-ethanol supernatant was incubated with $100 \%$ isopropanol for $10 \mathrm{~min}$ at room temperature. The mixture was then centrifuged again at $12,000 \mathrm{x} \mathrm{g}$ for $10 \mathrm{~min}$ at $4^{\circ} \mathrm{C}$ to pellet the protein. The protein pellet was washed twice with $0.3 \mathrm{M}$ guanidine hydrochloride and once with $100 \%$ ethanol. Then the protein pellet was solubilized in $1 \%$ SDS. For isolating protein from cells, the cells were first washed three times with cold PBS and lysed using radioimmunoprecipitation assay buffer on ice for $30 \mathrm{~min}$. The 
protein concentration was determined using a BCA assay (Merck KGaA). Protein samples $(40 \mu \mathrm{g})$ were separated by $12 \%$ SDS-PAGE and electro-transferred onto polyvinylidene fluoride membranes using the semi-dry transfer method. The membranes were blocked for $1 \mathrm{~h}$ with $5 \%$ non-fat milk at room temperature, and then were incubated at $4^{\circ} \mathrm{C}$ overnight with the following primary antibodies: Anti-FOXD1 (dilution, 1:500; cat. no. ab179940; Abcam, Cambridge, USA), anti-p21 (dilution, 1:1,000; cat. no. 2947S; CST Biological Reagents Co., Ltd., Shanghai, China), anti- $\beta$-actin (dilution, 1:5,000; cat. no. ab8226; Abcam). The membranes were subsequently washed with Tris-buffered saline with $0.1 \%$ Tween-20, and then incubated with the secondary horseradish peroxidase (HRP)-conjugated anti-mouse IgG antibody (dilution, 1:8,000; cat. no. 074-1806) or anti-rabbit IgG antibody (dilution, 1:6,000; cat no. 5220-0336) (both from Kirkegaard \& Perry Laboratories, Inc., Gaithersburg, MD, USA) for $2 \mathrm{~h}$ at room temperature. The signals were detected using an enhanced chemiluminescence system (GE Healthcare Life Sciences, Little Chalfont, UK). $\beta$-actin was used as an endogenous control.

Plasmid construction, lentivirus production and infection. For plasmid construction, the coding DNA sequence of FOXD1 was cloned into the pLenti-C-Myc-DDK-IRES-Puro tagged vector (PCMV; OriGene Technologies, Inc., Rockville, MD, USA). Short hairpin RNA (shRNA) targeting FOXD1 was purchased from Sigma-Aldrich; Merck KGaA, and cloned into the pLKO.1 vector (Addgene, Inc., Cambridge, MA, USA). A scrambled shRNA control (5'-CCTAAGGTTAAGTCGCCCTCGCTC GAGCGAGGGCGACTTAACCTTAGG-3') was cloned into the pLKO.1 vector and used as a negative control. The lentiviral packaging and envelope plasmids used were pMD2.G and psPAX2 (Addgene, Inc.). For lentivirus production, 293T cells were seeded at a density of $4 \times 10^{6}$ in $10 \mathrm{~cm}$ culture dishes and cultured for $16 \mathrm{~h}$. The PCMV vector and lentiviral packaging and envelope plasmids were co-transfected into 293T cells using Lipofectamine 3000 (Invitrogen; Thermo Fisher Scientific, Inc.). For stable transfection, cells (A2780, HO8910, SKOV3, CAOV3, OVCAR3 and H1299) were infected with lentivirus at a multiplicity of infection of 50 for $24 \mathrm{~h}$, and the cells were selected in medium containing puromycin $(2 \mu \mathrm{g} / \mathrm{ml})$ for 2 weeks.

Transfection of miRNA mimics and inhibitors. OVCAR3 cells were seeded in $6 \mathrm{~cm}$ dishes at a density of $5 \times 10^{5} / \mathrm{dish}$ at $24-36 \mathrm{~h}$ prior to transfection. Lipofectamine 3000 (Invitrogen; Thermo Fisher Scientific, Inc.) was employed to transfect the cells according to manufacturer's instructions. The concentration of miRNA mimics and inhibitors used were as follows: $80 \mathrm{nM}$ miR-30a-5p/miR-200a-5p mimics; $80 \mathrm{nM}$ mimics control; $100 \mathrm{nM}$ miR-30a-5p/miR-200a-5p inhibitors; and $100 \mathrm{nM}$ inhibitors control. The medium was refreshed at $6 \mathrm{~h}$ following transfection. The sequences of miRNA mimics and inhibitors was as follows: miR-30a-5p mimics, 5'-UGUAAACAUCCUC GACUGGAAG-3'; miR-30a-5p inhibitors, 5'-CUUCCAGUCG AGGAUGUUUACA; miR-200a-5p mimics, 5'-CAUCUUACC GGACAGUGCUGGA-3'; miR-200a-5p inhibitors, 5'-UCCAGC ACUGUCCGGUAAGAUG-3'; miRNA mimics negative control, 5'-UUCUCCGAACGUGUCACGUTT-3'; miRNA inhibitors negative control, 5'-CAGUACUUUUGUGUAGUA
CAA-3'. All the miRNA mimics and inhibitors were purchased from Guangzhou RiboBio Co., Ltd. (Guangzhou, China).

Small interfering (si)RNA transfection. A2780 cells $\left(2.5 \times 10^{5}\right)$ were plated in $6 \mathrm{~cm}$ dishes and transfected with p21 siRNA sequences using Lipofectamine 3000 according to the manufacturer's protocol (Invitrogen; Thermo Fisher Scientific, Inc.). At $48 \mathrm{~h}$ following transfection, the cells were harvested for MTT and clonogenic assay analysis. The sequences of the p21 siRNAs were the same as described previously $(38,39)$. The sequences of the p21 siRNA sequences were as follows: sip21-1, 5'-AGCGAU GGAACUUCGACUUTT-3'; sip21-2, 5'-AAUGGCGGGCUG CAUCCAGGATT-3'; siCtrl, 5'-UUCUCCGAACGUGUCACG UTT-3' (all purchased from Shanghai GenePharma Co., Ltd., Shanghai, China).

Clonogenic assay. Cells ( $\mathrm{n}=800-1,000 /$ well) stably expressing FOXD1 or transiently transfected with p21 siRNA and FOXD1 were plated in 6-well plates and cultured for 2-3 weeks. The colonies were fixed with $100 \%$ methanol for $15 \mathrm{~min}$ at room temperature and then stained with $1 \%$ crystal violet for $10 \mathrm{~min}$ at room temperature. The number of colonies was assessed using ImageQuant TL software (version 8.1; GE Healthcare Life Sciences). The data are presented as the mean \pm standard error of three independent experiments.

Cell proliferation assay. Cell proliferation was assessed using an MTT assay. A2780 cells (800 cells/well) were first seeded in triplicate wells of a 96 -well plate. MTT reagent $(5 \mathrm{mg} / \mathrm{ml}$; cat no. M2128; Sigma-Aldrich; Merck KGaA) was subsequently added to the wells and incubated for $4 \mathrm{~h}$. DMSO was subsequently added to dissolve the formazan crystals and the absorbance at $490 \mathrm{~nm}$ was then read.

Dual-luciferase reporter assay. In order to investigate whether FOXD1 may be a direct target of miR-30a-5p and miR-200-5p, the 3'-UTR of the FOXD1 mRNA sequence containing the putative miR-30a-5p and miR-200a-5p binding sites were cloned into the pmirGLO Dual-Luciferase miRNA Target Expression Vector (Promega Corporation, Madison, WI, USA). The FOXD1 mutant sequences were constructed by Sangon Biotech, Co., Ltd. (Shanghai, China) and cloned into pmirGLO vector. 293T cells were co-transfected with miR-30a-5p or miR-200a-5p mimics and the pmirGLO vector containing either the wild-type or mutant FOXD1 sequence using Lipofectamine 3000 according to the manufacturer's protocol (Invitrogen; Thermo Fisher Scientific, Inc.). At $48 \mathrm{~h}$ following transfection, luciferase activity was measured using the Dual-Glo Luciferase Assay System (Promega Corporation).

To investigate FOXD1 binding sites in the p21 promoter, three predicted wild-type binding sites of FOXD1 were separately cloned into the pGL4.26[luc $2 / \mathrm{minP} /$ Hygro] vector (Promega Corporation), and the mutant binding sites were constructed by Sangon Biotech, Co., Ltd. and cloned into the pGL4.26 vector. The pGL4.74[hRluc/TK] vector (Promega Corporation) was used as an endogenous control. 293T cells (4x10 $/$ well) were seeded in a 96-well plate and transfected with vectors using Lipofectamine 3000 according to the manufacturer's protocol (Invitrogen; Thermo Fisher Scientific, Inc.). Cells were transfected with vectors at the following 
concentration ratio: PCMV (75 ng): pGL4.26 (25 ng): pGL4.74 $(2.5 \mathrm{ng})$. At $48 \mathrm{~h}$ following transfection, luciferase activity was measured.

Immunohistochemical staining. The TMA of HGSOC samples were incubated at $65^{\circ} \mathrm{C}$ for $30 \mathrm{~min}$ and deparaffinized in xylene immediately. The TMA was subsequently rehydrated in a graded ethanol series. Antigen retrieval was performed in citric acid buffer ( $\mathrm{pH}$ 6.0) by heat treatment $\left(98^{\circ} \mathrm{C}\right)$ for $15 \mathrm{~min}$. This assay was performed using the SP-9000 IHC reagent kit (cat. no. SP-9000; OriGene Technologies, Inc.). The samples were blocked in $1.5 \%$ goat serum from the SP-9000 IHC reagent kit at $37^{\circ} \mathrm{C}$ for $20 \mathrm{~min}$, and the TMA was then incubated with primary antibodies against FOXD1 (dilution, 1:1,000; cat. no. ab179940; Abcam) at $4^{\circ} \mathrm{C}$ for $14 \mathrm{~h}$. The TMA was subsequently washed and incubated with a HRP-conjugated secondary antibody from the SP-9000 IHC reagent kit (OriGene Technologies, Inc.) for $30 \mathrm{~min}$ at $37^{\circ} \mathrm{C}$. Staining was detected using a 3,3'-diaminobenzidine detection system. The Eclipse Ni-E microscope (Nikon Corporation, Tokyo, Japan) was used to visualize the stained tissue sections. As transcription factors modulate gene expression by binding to the promoter of target genes in the nucleus, the expression of FOXD1 in the nucleus was determined in the present study (40-42). The final score of each sample was decided by two pathologists and was dependent on the extent and intensity of staining. The intensity of staining was evaluated and scored as follows: 0 , negative; 1 , weak; 2 , moderate; or 3 , strong. The proportion of positively stained cells was divided into four levels and scored as follows: $1,0-25 \% ; 2,26-50 \% ; 3,51-75 \%$; and $4,76-100 \%$. Staining scores for FOXD1 were calculated by multiplying the score assigned to the intensity of staining $(0-3)$ by the score assigned to the percentage of positively stained cells (1-4). According to the staining scores, the samples were divided into the low expression group (score $<6$ ) and high expression group (score $\geq 6$ ). For the immunohistochemical staining of xenograft tumor samples from mice, the tumor tissues were fixed in $10 \%$ formalin for 2 days at room temperature and then embedded in paraffin. The samples were then sectioned (4- $\mu \mathrm{m}$ thickness), and then incubated at $65^{\circ} \mathrm{C}$ for $30 \mathrm{~min}$ before they were deparaffinized and rehydrated in a graded ethanol series. For antigen retrieval, the tissue sections were immersed in the citric acid buffer $(\mathrm{pH} 6.0)$ and incubated at $98^{\circ} \mathrm{C}$ for $15 \mathrm{~min}$. The samples were blocked in $1.5 \%$ goat serum (SP-9000 IHC reagent kit; OriGene Technologies, Inc.) at $37^{\circ} \mathrm{C}$ for $20 \mathrm{~min}$. The tissue sections were then incubated with primary antibodies against FOXD1 (dilution, 1:1,000; cat. no. ab179940; Abcam) and p21 (dilution, 1:1,000; cat. no. 2947S; CST Biological Reagents Co., Ltd.) at $4^{\circ} \mathrm{C}$ for $14 \mathrm{~h}$. The sections were washed and incubated with a HRP-conjugated secondary antibody from the SP-9000 IHC reagent kit (OriGene Technologies, Inc.) for $30 \mathrm{~min}$ at $37^{\circ} \mathrm{C}$. Staining was detected using a 3,3'-diaminobenzidine detection system.

Cell cycle synchronization and analysis. Cells (A2780, HO8910, SKOV3, OVCAR3, CAOV3 and H1299) stably overexpressing FOXD1 were seeded in $6 \mathrm{~cm}$ dishes at a density of $1.2-2.0 \times 10^{5}$ cells/well and cultured until they were $30-40 \%$ confluent. The cells were then synchronized using a double thymidine block as described previously (43) in order to synchronize cells in G1 phase. The cells were then released from this phase at different intervals (A2780, $3 \mathrm{~h} ; \mathrm{HO} 8910,6 \mathrm{~h}$; SKOV3, 12 h; OVCAR3, 6 h; CAOV3, 12 h; H1299, 10 h) by replacing the medium with complete culture medium without thymidine. The cells were harvested for cell cycle analysis using the BD FACSCalibur Flow Cytometer (BD Biosciences, Franklin Lakes, NJ, USA) and the results were analyzed using ModFit LT software (version 2.0; Verity Software House, Topsham, ME, USA).

Chromatin immunoprecipitation (ChIP) assay. A ChIP assay was performed to enrich the specific regions of chromatin immunoprecipitated by a specific antibody via using the ChIP-IT Express Chromatin Immunoprecipitation kit (Active Motif, Carlsbad, CA, USA) according to the manufacturer's protocol. A2780 cells $\left(1 \times 10^{7}\right)$ stably overexpressing FOXD1 were first cross-linked and sheared into 500-bp DNA fragments by sonication. The DNA fragments were then selectively immunoprecipitated from the cell debris by incubating with a DYKDDDDK Tag antibody (dilution, 1:50; cat. no. 14793S), or a rabbit IgG antibody (dilution, 1:50; cat. no. 2729) (both from CST Biological Reagents Co., Ltd.), which was used as negative control, for $16 \mathrm{~h}$ at $4^{\circ} \mathrm{C}$. The purified DNA was amplified by PCR using the Phusion High-Fidelity PCR Master Mix with HF Buffer (cat no. M0531; New England Biolabs, Inc., Ipswich, MA, USA) and the following primers: Site B, forward, 5'-GTCGTGGTGGTGGTGA-3' and reverse, 5'-CTGCTTTC AGGCATTTC-3'; site C, forward, 5'-ATGTCATCCTCCTGA TCTTT-3' and reverse, 5'-AGTCCCTCGCCTGCGTTGGT-3'. The thermal cycling parameters were as follows: $30 \mathrm{sec}$ at $98^{\circ} \mathrm{C}$ followed by 40 cycles at $98^{\circ} \mathrm{C}$ for $10 \mathrm{sec}, 60^{\circ} \mathrm{C}$ for $30 \mathrm{sec}$, $72^{\circ} \mathrm{C}$ for $15 \mathrm{sec}$, and then a final step at $72^{\circ} \mathrm{C}$ for $6 \mathrm{~min}$. The PCR products were separated by $2 \%$ agarose gel electrophoresis and stained with ethidium bromide.

In vivo studies. All experiments involving animals were approved by the Ethics Committee on Animal Experiments of Shandong University. A total of $20 \mathrm{BALB} / \mathrm{c}$ nu/nu female mice (age, 6-7 weeks; weight, $20.3 \pm 1.5 \mathrm{~g}$ ) were maintained in a pathogen-free facility (temperature, $25^{\circ} \mathrm{C}$; humidity, $50-60 \% ; 12 \mathrm{~h} \mathrm{light/day} \mathrm{cycles)} \mathrm{and} \mathrm{had} \mathrm{free} \mathrm{access} \mathrm{to} \mathrm{water}$ and food. The mice were equally divided into A2780 and OVCAR3 groups, and each group was further subdivided into a control and FOXD1 overexpression group. For the in vivo xenograft experiments, A2780 and OVCAR3 cells transfected with PCMV or PCMV-FOXD1 vectors $\left(6 \times 10^{6}\right.$ cells) were injected subcutaneously into the right flank region of the mice. Following 4 weeks (A2780 cells) or 5 weeks (OVCAR3 cells), the mice were sacrificed to determine tumor weight and volume. Tumor volume was calculated using the following formula: Volume $=\left[(\right.$ long diameter $) \times$ (short diameter) $\left.{ }^{2}\right] \times 1 / 2$. Immunohistochemical staining was employed for the detection of FOXD1 and p21 using the aforementioned methods.

Bioinformatics analyses. The GSE9891 dataset (https://www. ncbi.nlm.nih.gov/geo/query/acc.cgi?acc=GSE9891) contains gene expression data for 285 ovarian carcinoma samples (44). Following the removal of 7 samples without corresponding clinical survival information, and two samples derived from patients that succumbed due to unrelated causes, the remaining 276 samples were included in the current study. 
The inclusion criteria for HGSOC were as follows: Serous histological subtype and a pathological grade of 3. A total of 152 patients were identified and divided into high and low FOXD1 expression groups. The significance cut-off value was calculated using the X-Tile (version 3.6.1) bioinformatics tool (45). To identify predicted miRNA sequences that may regulate FOXD1, TargetScan (http://www.targetscan.org/) and miRanda (http://www.microrna.org) were employed $(46,47)$. A total of 142 candidate miRNAs binding sites were identified by TargetScan (context, ++; score >90; version 7.1) and 146 candidate miRNA binding sites were identified by miRanda (version: August 2010 Release; mirSVR score $\leq-0.1$ ). To identify target genes that may be regulated by FOXD1, MatInspector (version 8.4; http://www.genomatix.de) and JASPAR (version 2016; http://jaspar.genereg.net) were employed $(48,49)$. A region $2 \mathrm{~kb}$ upstream of target gene promoters was screened for FOXD1-binding sites.

Statistical analysis. Data are expressed as the mean \pm standard deviation of three independent experiments. Overall survival and progression-free survival analysis was performed using Kaplan-Meier plots and a log-rank test. A Student's t-test was selected to analyze significant differences between two groups. Analysis of variance and the Dunnett's post hoc test was used to compare the means among $>2$ groups. The $\chi^{2}$ test was used to compare clinical characteristics. $\mathrm{P}<0.05$ was considered to indicate a statistically significant difference.

\section{Results}

FOXD1 is downregulated in HGSOC and elevated FOXD1 predicts good prognosis. To determine the protein and mRNA expression levels of FOXD1 in HGSOC tissue samples and normal FT samples, western blot and RT-qPCR analyses were performed, respectively. At the protein level, the expression of FOXD1 was higher in FT (n=7) when compared with HGSOC $(\mathrm{n}=8)$ samples (Fig. 1A). At the mRNA level, the expression of FOXD1 was significantly higher in FT $(\mathrm{n}=11)$ compared with HGSOC $(n=20)$ samples (Fig. 1B). To further confirm the role of FOXD1 in HGSOC, immunohistochemical staining was performed to measure its expression in the HGSOC TMA $(n=120)$. As expected, the FOXD1 transcription factor was primarily located in the nucleus (Fig. 1C). The stained HGSOC tissue samples were scored and divided into high $(n=58)$ and low ( $n=62)$ FOXD1 expression groups. The overall survival rate of the high expression group was significantly higher when compared with the low expression group ( $\mathrm{P}=0.018$; Fig. 1D). The association between FOXD1 expression and clinicopathological parameters was also analyzed; however, no statistically significant associations were identified (Table I). To investigate these observations further, the GSE9891 dataset was used to evaluate the prognostic role of FOXD1 at the mRNA level (44). The expression of FOXD1 in HGSOC samples alone was first determined. As demonstrated in Fig. 1E, the overall survival $(\mathrm{P}=0.030)$ and progression-free survival $(\mathrm{P}=0.003)$ of the low FOXD1 expression group was significantly reduced when compared with the high FOXD1 expression group. Samples of 276 in the GSE9891 cohort were then divided into high and low FOXD1 expression groups. As shown in Fig. 1F, the overall survival $(\mathrm{P}=0.010)$ and $\mathrm{PFS}(\mathrm{P}=0.011)$ of the low FOXD1
Table I. Association between FOXD1 expression and the clinicopathological features of patients with high-grade serous ovarian carcinoma.

\begin{tabular}{|c|c|c|c|}
\hline \multirow{2}{*}{$\begin{array}{l}\text { Clinicopathological } \\
\text { feature }\end{array}$} & \multicolumn{2}{|c|}{ FOXD1 expression } & \multirow[b]{2}{*}{ P-value } \\
\hline & Low & High & \\
\hline \multicolumn{4}{|l|}{ Age (years) } \\
\hline$<55$ & 29 & 33 & \multirow[t]{2}{*}{0.724} \\
\hline$\geq 55$ & 29 & 29 & \\
\hline \multicolumn{4}{|l|}{ FIGO stage } \\
\hline I-II & 14 & 13 & \multirow[t]{2}{*}{0.983} \\
\hline III-IV & 48 & 45 & \\
\hline \multicolumn{4}{|l|}{ CA125 (U/ml) } \\
\hline$<600$ & 22 & 18 & \multirow[t]{2}{*}{0.605} \\
\hline$\geq 600$ & 40 & 40 & \\
\hline \multicolumn{4}{|l|}{ Platinum status } \\
\hline Sensitive & 27 & 25 & \multirow[t]{2}{*}{0.099} \\
\hline Resistant & 2 & 7 & \\
\hline \multicolumn{4}{|l|}{ Residual lesions } \\
\hline$<1$ & 39 & 37 & \multirow[t]{2}{*}{0.903} \\
\hline$\geq 1$ & 21 & 19 & \\
\hline
\end{tabular}

FOXD1, forkhead box D1; FIGO, Fédération Internationale de Gynécologie et d'Obstétrique; CA125, cancer antigen 125.

expression group was significantly reduced when compared with the high FOXD1 expression group. These results provide evidence to suggest that reduced FOXD1 expression may predict poor prognosis in HGSOC patients.

FOXD1 influences the proliferation of ovarian cancer and H1299 cells. To investigate the effect of FOXD1 expression on ovarian cancer cells, five ovarian cancer cell lines, including A2780, HO8910, SKOV3, OVCAR3, CAOV3 and one lung cancer cell line, H1299, were employed and transfected with a FOXD1 overexpression vector or an shRNA targeting FOXD1. As demonstrated in Fig. 2A, overexpression of FOXD1 significantly inhibited the colony-forming efficiency of all six cell lines when compared with empty vector controls. By contrast, downregulation of FOXD1 significantly increased their clonogenicity compared with an empty vector control (Fig. 2A). The cell cycle distribution among these transfected cell lines was then measured. For cell cycle analysis, the cells were synchronized in G1 phase using the double thymidine block method. Then the cells were released by replacing the medium with thymidine-free complete culture medium. As shown in Fig. 2B, upregulated FOXD1 expression induced cell cycle arrest at the G0/ G1 phase and downregulation of FOXD1 reversed this effect. The results indicate that FOXD1 may suppress the proliferation of ovarian cancer cells by inducing cell cycle arrest in G1 phrase in vitro.

FOXD1 inhibits tumor growth in vivo. The Cancer Genome Atlas Research Network reported that HGSOC is 
A

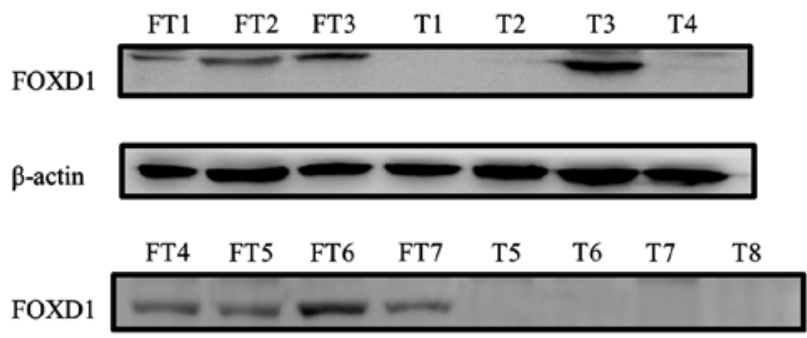

$\beta$-actin
B

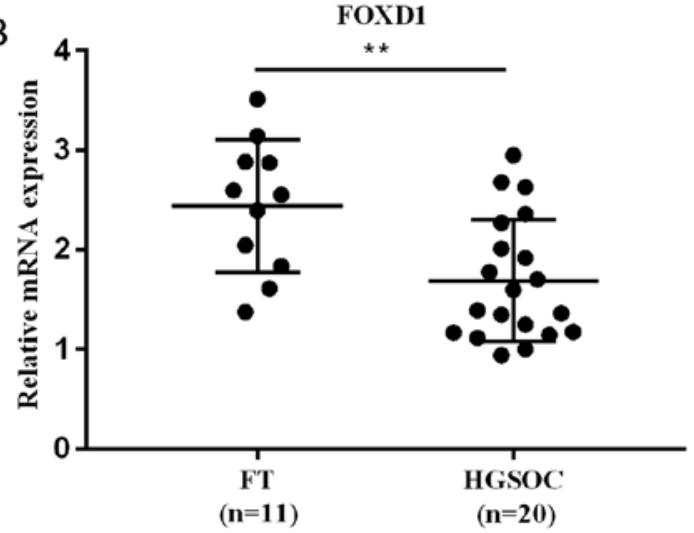

$\mathrm{C}$
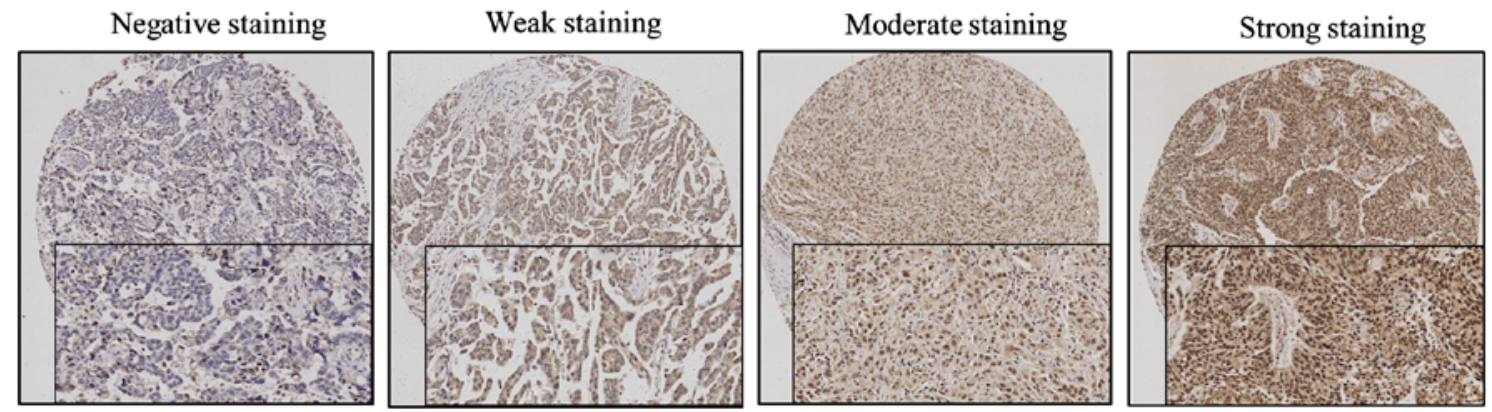

$\mathrm{D}$

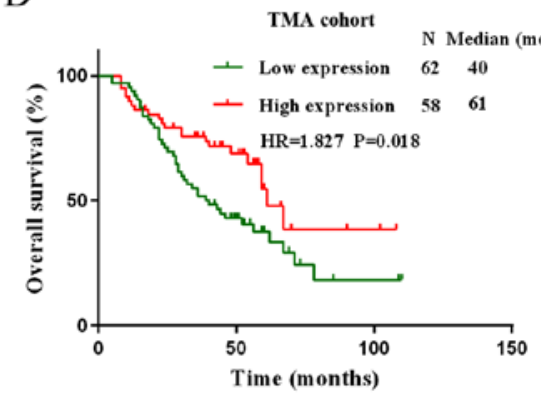

E
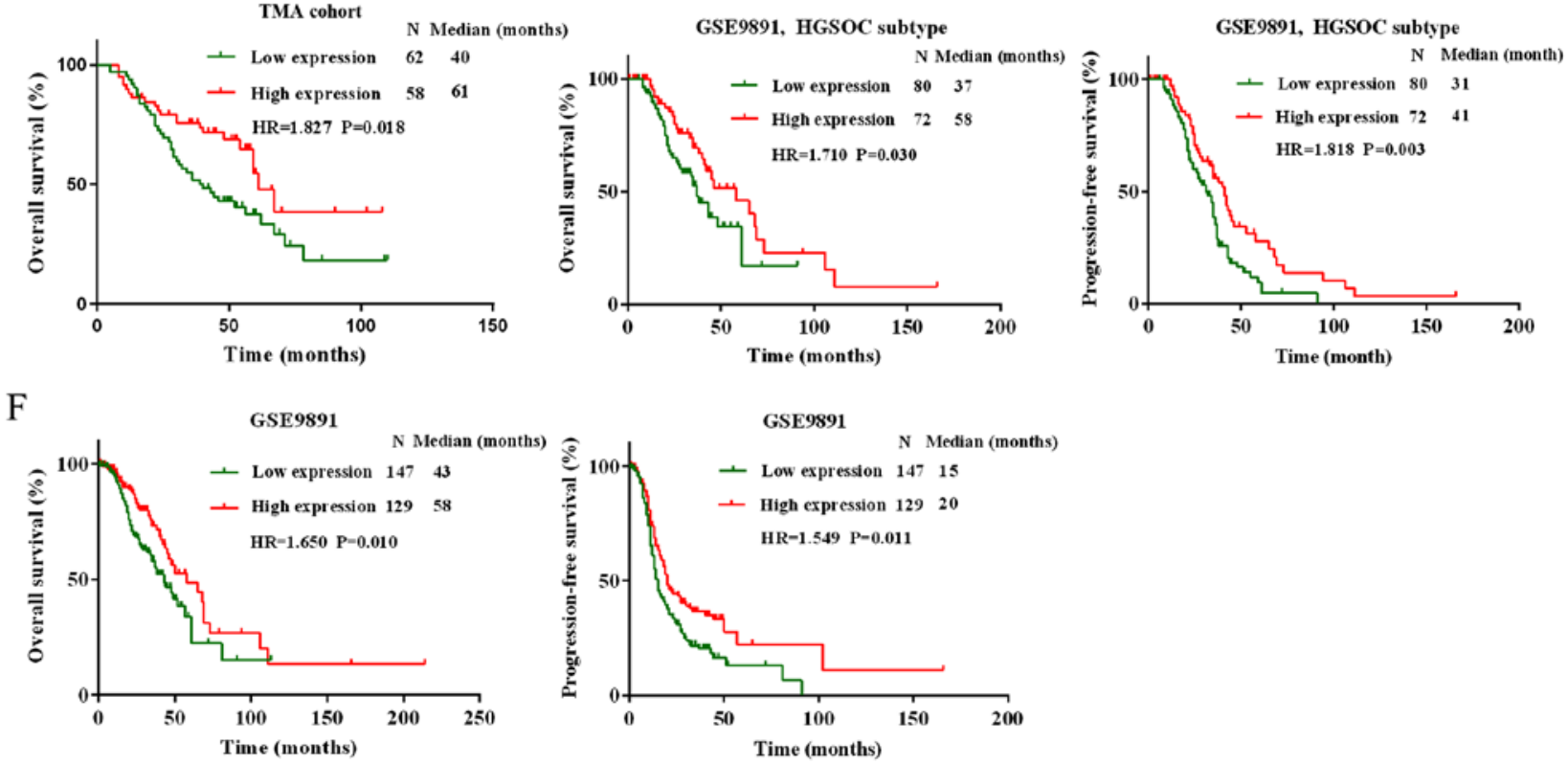

Figure 1. Expression of FOXD1 in HGSOC. (A) The protein expression levels of FOXD1 in HGSOC samples (n=8) and normal FT samples (n=7). (B) The mRNA levels of FOXD1 in HGSOC samples $(n=20)$ and normal FT samples $(n=11)$. (C) Representative immunohistochemical staining images of negative, weak, moderate and strong FOXD1 expression in the TMA cohort (magnification, x100). (D) Kaplan-Meier curves demonstrating overall survival rates of patients with low and high FOXD1 expression in the TMA cohort. (E) Overall survival and progression-free survival of patients with HGSOC from the GSE9891 cohort, divided into high and low FOXD1 expression groups. (F) Overall survival and progression-free survival of 276 ovarian cancer patients from the GSE9891 cohort, divided into high and low FOXD1 expression groups. ${ }^{* *} \mathrm{P}<0.01$, as indicated. FOXD1, forkhead box D1; HGSOC, high-grade serous ovarian carcinoma; FT, fallopian tube; TMA, tissue microarray; T, HGSOC tumor samples.

characterized by TP53 mutations in $~ 96 \%$ of tumors (50). To further validate the role of FOXD1 in vivo, OVCAR3 (p53 mutant) and A2780 (p53 wild-type) cells with or without FOXD1 overexpression, were subcutaneously inoculated into nude mice ( $\mathrm{n}=5$ per group). Following $4-5$ weeks, the mice were sacrificed, and the tumor weight and volume were measured. As demonstrated in Fig. 3A and B, the weight and volume of tumors from mice in the FOXD1 overexpression group was significantly lower when compared with the control group for both cell lines. Immunohistochemical staining of the xenograft tumor tissues was then performed to examine the expression of FOXD1 and p21. As shown in Fig. 3C, the expression of FOXD1 in nucleus was positively associated with $\mathrm{p} 21$ expression in the nucleus. The results indicate that 
A
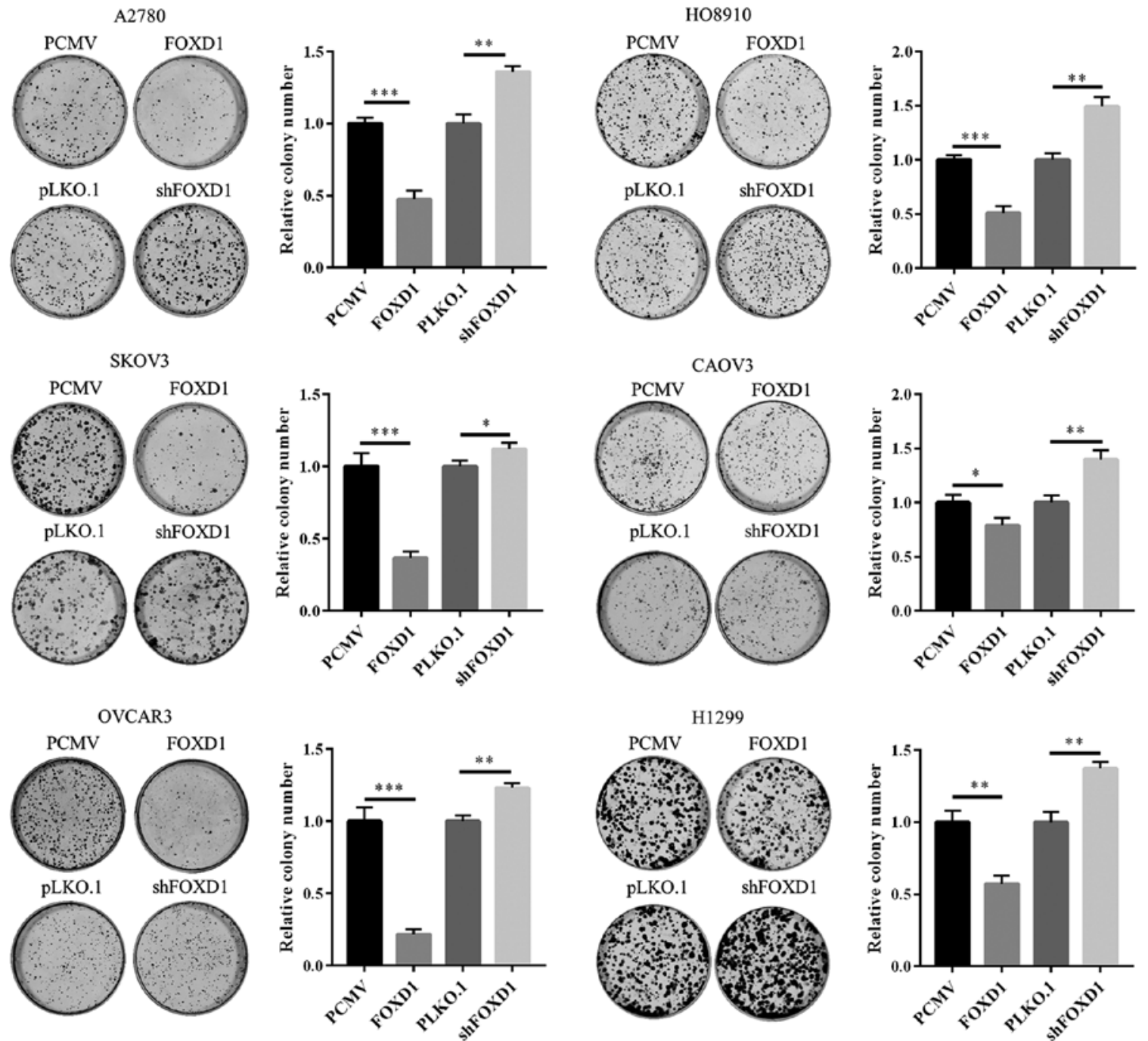

B
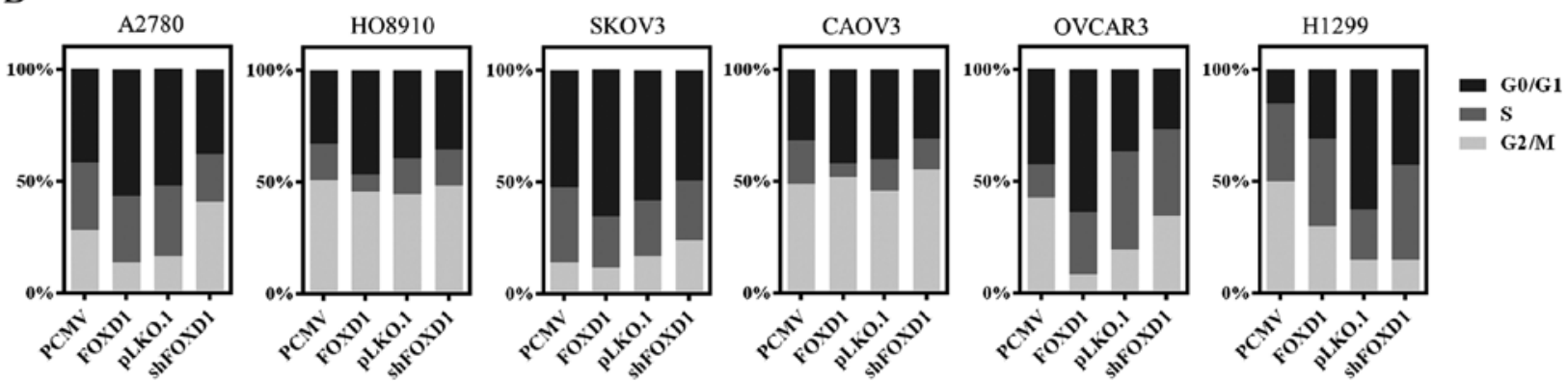

Figure 2. FOXD1 inhibits ovarian cancer cell proliferation in vitro. (A) A clonogenic assay was employed to measure the effect of FOXD1 on the proliferation of five ovarian cancer cell lines (A2780, HO8910, SKOV3, CAOV3 and OVCAR3) and the p53-null H1299 lung cancer cell line. (B) The six cancer cell lines were first synchronized and then released for cell cycle analysis by flow cytometry. The results are expressed as mean \pm standard deviation. ${ }^{*} \mathrm{P}<0.05,{ }^{* * *} \mathrm{P}<0.01$ and ${ }^{* * * *} \mathrm{P}<0.001$ as indicated. FOXD1, forkhead box D1; PCMV, pLenti-C-Myc-DDK-IRES-Puro tagged vector; shFOXD1, short hairpin RNA targeting FOXD1

FOXD1 may inhibit the proliferation of ovarian cancer cells in vivo.

miR-30a-5p and miR-200a-5p are direct regulators of FOXDI expression. From the results presented thus far, the authors hypothesized that FOXD1 may function as a tumor suppressor in ovarian cancer. To investigate the molecular mechanisms underlying the downregulation of FOXD1 expression in ovarian cancer, miRNA target prediction tools (miRanda and TargetScan) were used to identify putative miRNA sequences that may regulate FOXD1. Among all candidate miRNAs identified, miR-30a-5p and miR-200a-5p were selected for further investigation. According to previous studies, miR-30a-5p and miR-200a-5p have been observed to be upregulated in ovarian cancer (30-36). As demonstrated in Fig. 4A and B, the expression levels of miR-30a-5p and miR-200a-5p were determined in HGSOC and normal FT tissue samples. HGSOC samples were observed to exhibit significantly higher levels of miR-30a-5p and miR-200a-5p expression when compared with normal FT samples (Fig. 4A and B). A dual-luciferase reporter 
A

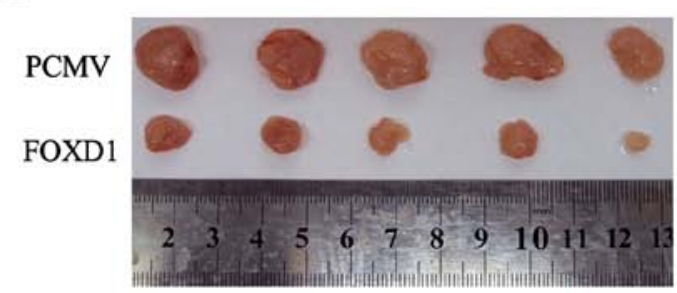

B

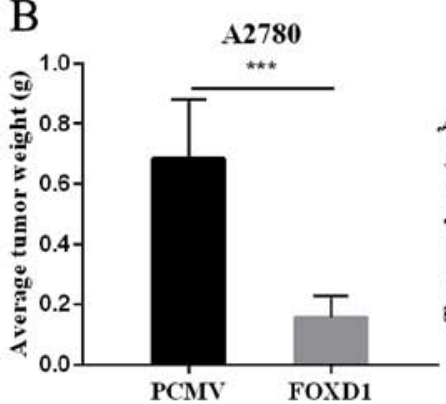

C
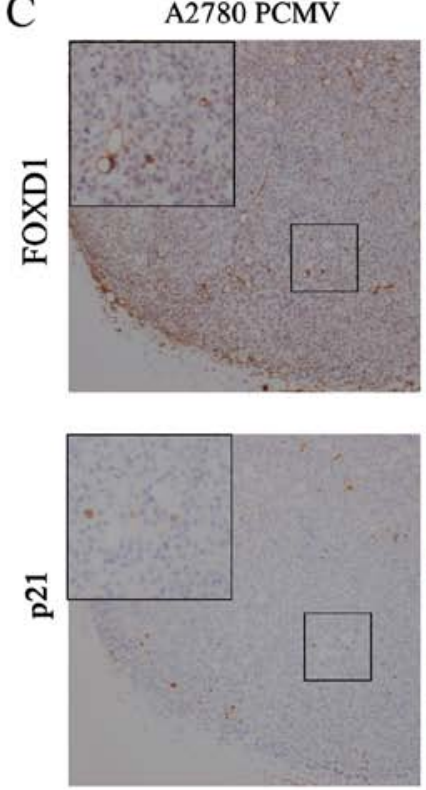

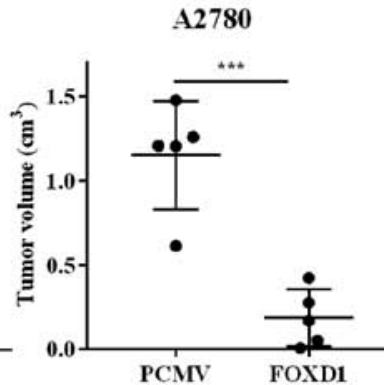

A2780 FOXD1
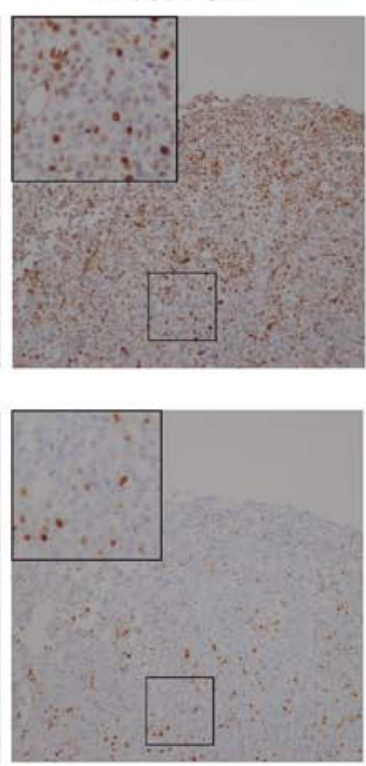
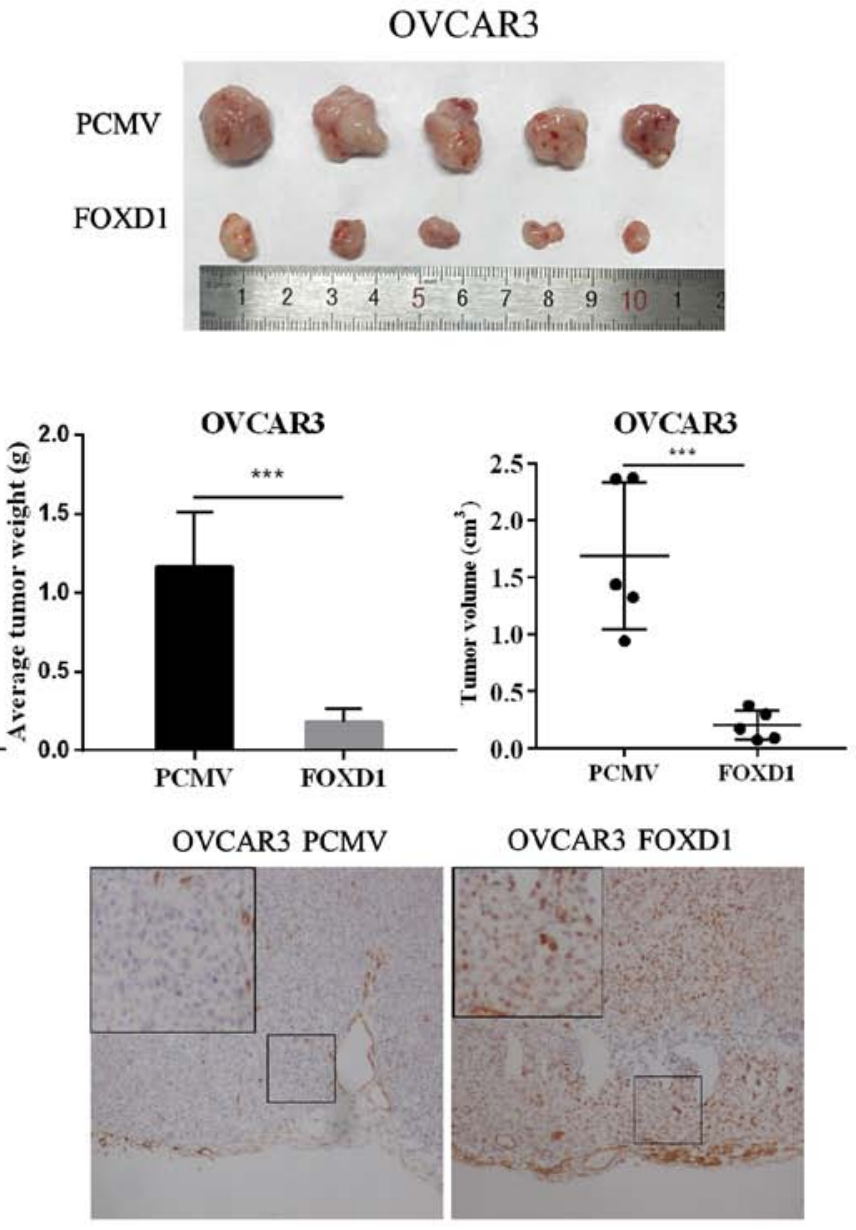

OVCAR3 FOXD1
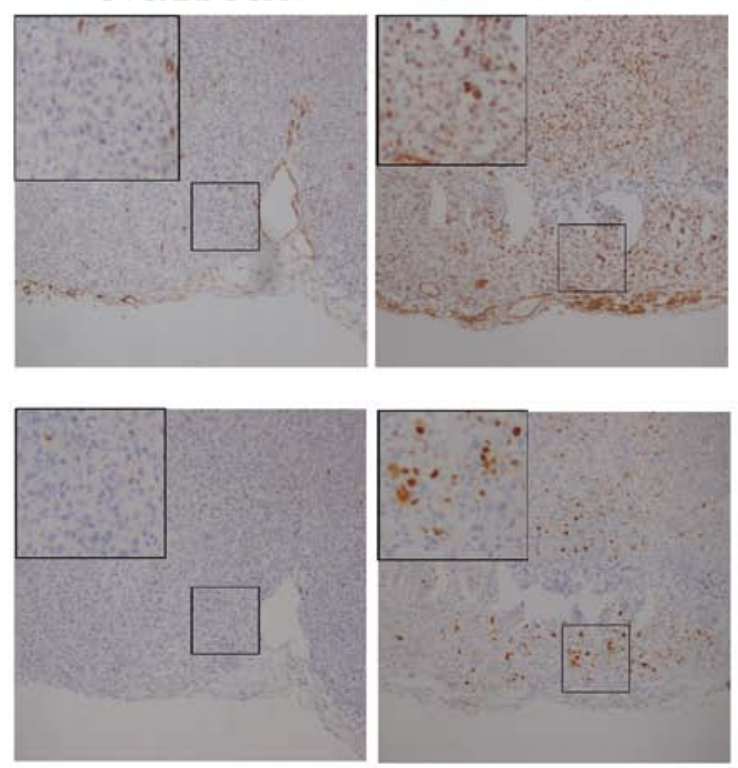

Figure 3. FOXD1 inhibits tumor growth in vivo. (A) A2780 and OVCAR3 cells with or without FOXD1 overexpression were subcutaneously inoculated into nude mice (n=5 mice/group). (B) Following 4-5 weeks, the mice were sacrificed for assessment of tumor weight and volume. (C) Representative immunohistochemistry staining images showing FOXD1 and p21 expression in xenograft tumor tissues obtained from the mice (magnification, x100). Data are expressed as the mean \pm standard deviation. ${ }^{* * *} \mathrm{P}<0.001$, as indicated. FOXD1, forkhead box D1; PCMV, pLenti-C-Myc-DDK-IRES-Puro tagged vector.

assay was subsequently employed to verify the binding sites for miR-30a-5p and miR-200a-5p in the FOXD1 3'-UTR region. Wild-type or mutant FOXD1 3'-UTR sequences were cloned into the luciferase vector, and then co-transfected with miR-30a-5p or miR-200a-5p mimics into $293 \mathrm{~T}$ cells Upregulation of miR-30a-5p or miR-200a-5p significantly decreased the luciferase activity of cells transfected with wild-type but not the mutant FOXD1 vector (Fig. 4C and D). The role of miR-30a-5p and miR-200a-5p in ovarian cancer cells was then investigated. OVCAR3 cells were transfected with miR-30a-5p and miR-200a-5p mimics or inhibitors. As shown in Fig. 4E and F, the expression of FOXD1 at mRNA and protein levels demonstrated a positive association with p21 expression. These results indicated that miR-30a-5p and miR-200a-5p may directly downregulate FOXD1 expression.
FOXD1 directly promotes p 21 expression in vitro and in vivo. A region $2 \mathrm{~kb}$ upstream of the $\mathrm{p} 21$ promoter was screened for FOXD1-binding sites using online promoter prediction tools such as MatInspector and JASPAR. FOXD1 was identified as a putative regulator of $\mathrm{p} 21$ by JASPAR. A total of three candidate transcription factor binding sites (TFBSs) were also identified (Fig. 5A). A dual-luciferase reporter assay was employed to verify these TFBSs. As shown in Fig. 5B, two out of the three TFBSs in the FOXD1 overexpression group (sites B and C) demonstrated a significant increase in luciferase activity compared with the empty vector controls. When sites $\mathrm{B}$ and $\mathrm{C}$ were mutated, no significant difference in luciferase activity was observed between the FOXD1 overexpression group and control group (Fig. 5C). A ChIP assay and PCR were subsequently performed to further verify the 
A

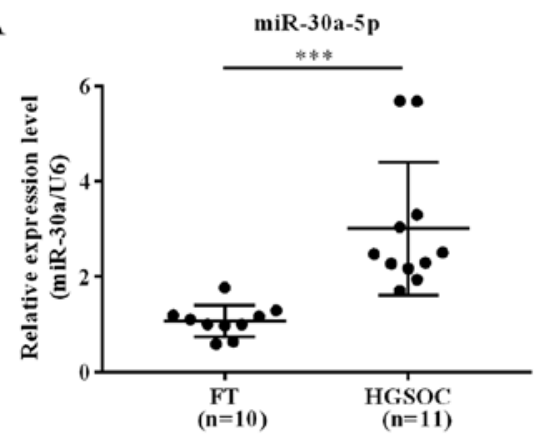

$\mathrm{C}$

$\mathrm{D}$

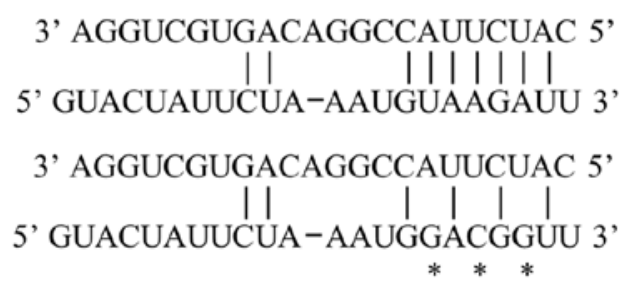

$\mathrm{B}$

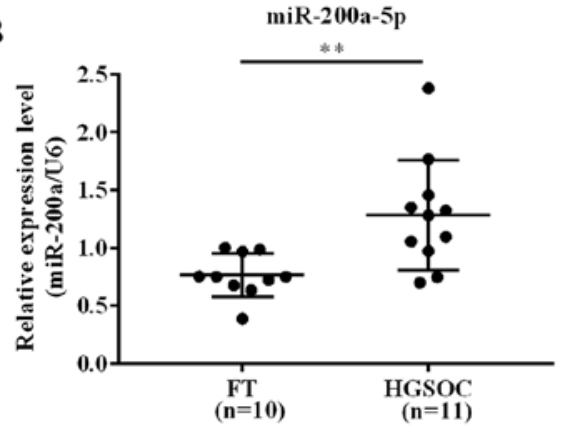

miR-30a-5p

FOXD1 (WT)

miR-30a-5p

FOXD1 (MUT)
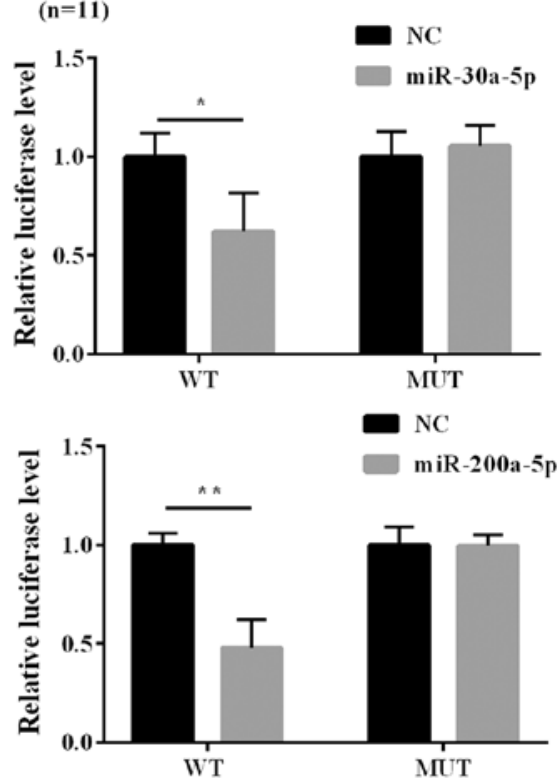

E

miR-200a-5p

FOXD1 (WT)

miR-200a-5p

FOXD1 (MUT)

miR-30a-5p
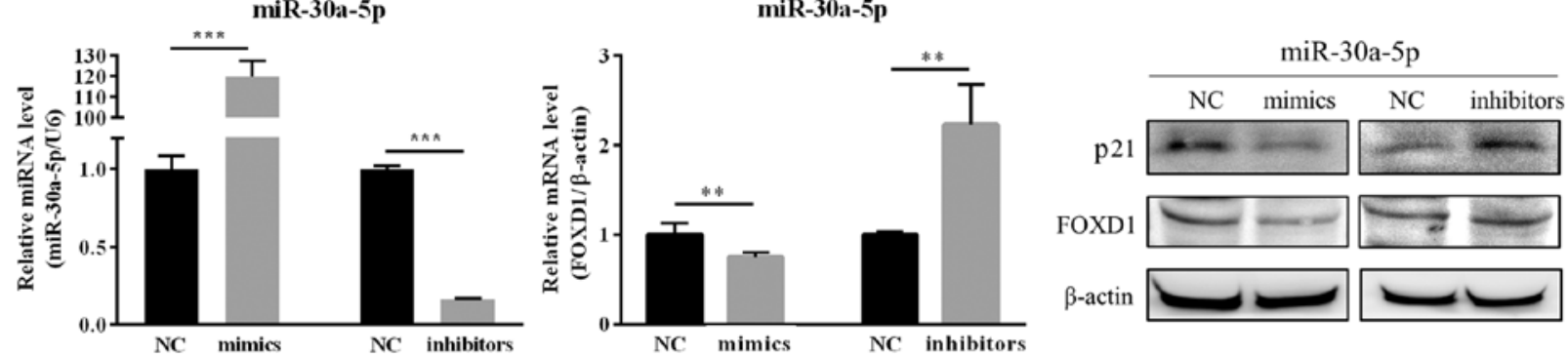

$\mathrm{F}$
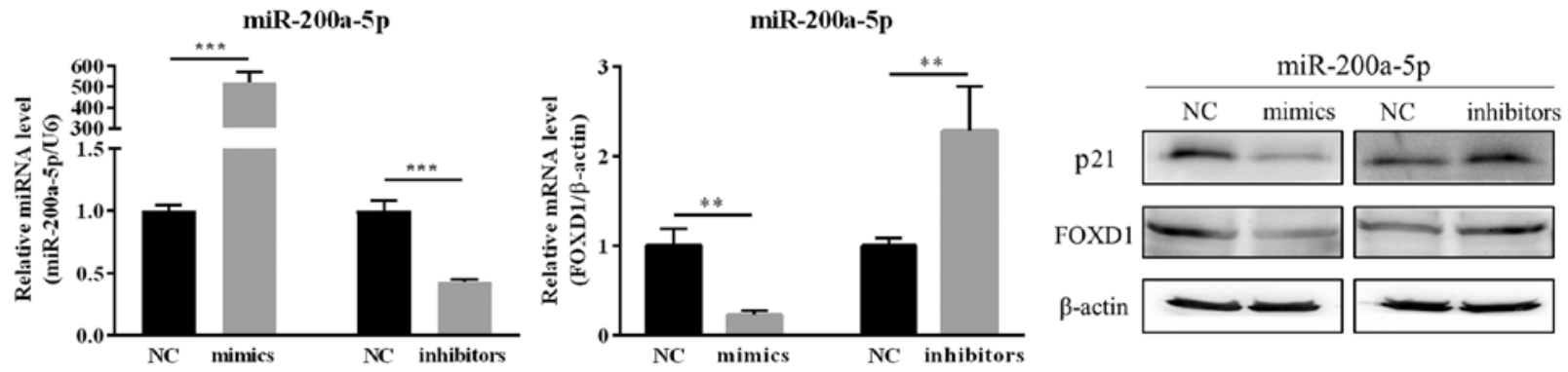

Figure 4. Expression of miR-30a-5p and miR-200a-5p is upregulated in HGSOC, and these miRNAs directly target FOXD1 via predicted binding sites. RT-qPCR analysis demonstrated that (A) miR-30a-5p and (B) miR-200a-5p were upregulated in HGSOC (n=11) compared with normal FT samples (n=10). Schematics indicating the predicted binding sites of (C) miR-30a-5p and (D) miR-200a-5p in the 3'-UTR of FOXD1 mRNA. Asterisks indicate the mutated binding sites. Reporter vectors with wild-type or mutant FOXD1 3'-UTRs were co-transfected with miR-30a-5p/miR-200a-5p mimics in 293T cells and the relative luciferase activities are shown. The transfection efficiency was examined by RT-qPCR. Western blotting and RT-qPCR were employed to evaluate the expression of p21 and FOXD1 in OVCAR3 cells transfected with (E) miR-30a-5p mimics and inhibitors and (F) miR-200a-5p mimics and inhibitors. The results are expressed as the mean \pm standard deviation. ${ }^{*} \mathrm{P}<0.05,{ }^{* *} \mathrm{P}<0.01$ and ${ }^{* * *} \mathrm{P}<0.001$, as indicated. miR, microRNA, FOXD1, forkhead box D1; HGSOC, high-grade serous ovarian carcinoma; RT-qPCR, reverse transcription, quantitative polymerase chain reaction; FT, fallopian tube; UTR, untranslated region; WT, wild-type; MUT, mutant; NC, negative control.

TFBSs. As shown in Fig. 5D, enrichment of the p21 promoter fragments were confirmed by PCR using primers flanking
FOXD1 binding sites (sites B and C). The expression of p21 in five ovarian cancer cell lines and one lung carcinoma cell 
A

\begin{tabular}{c}
\multicolumn{1}{c}{$\begin{array}{c}\text { Site } \mathrm{A} \\
\mathrm{-1901}\end{array}$} \\
\hline
\end{tabular}

B
WT

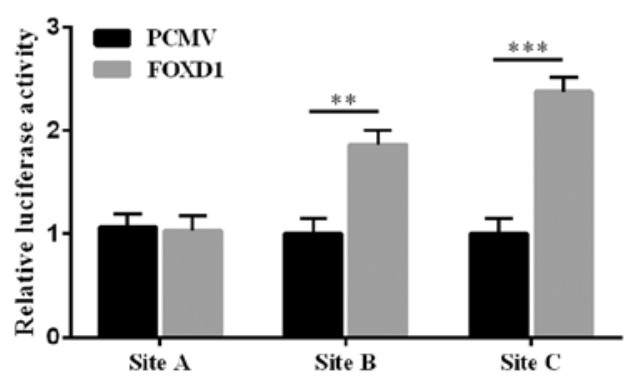

$\mathrm{C}$

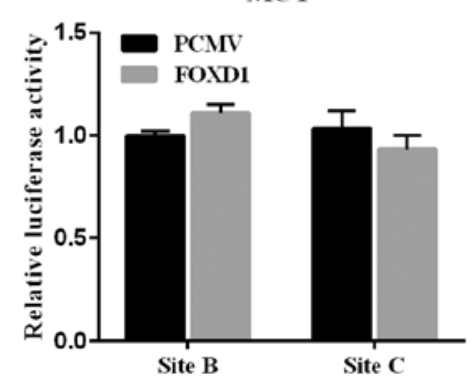

D

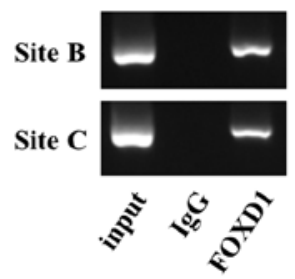

SKOV3

E

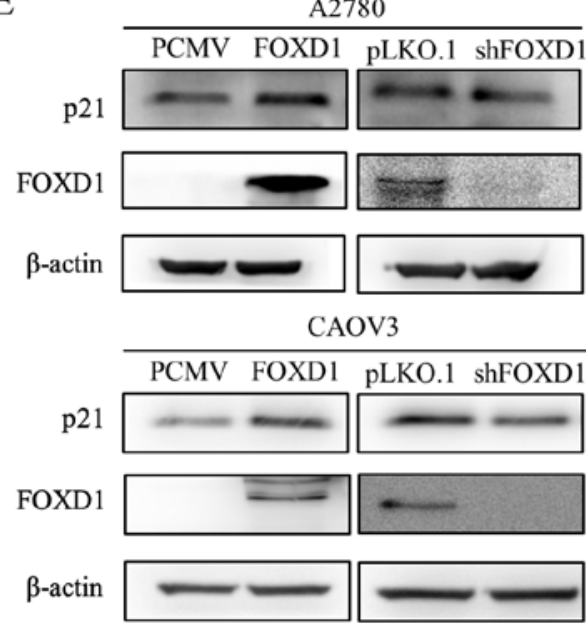

HO8910

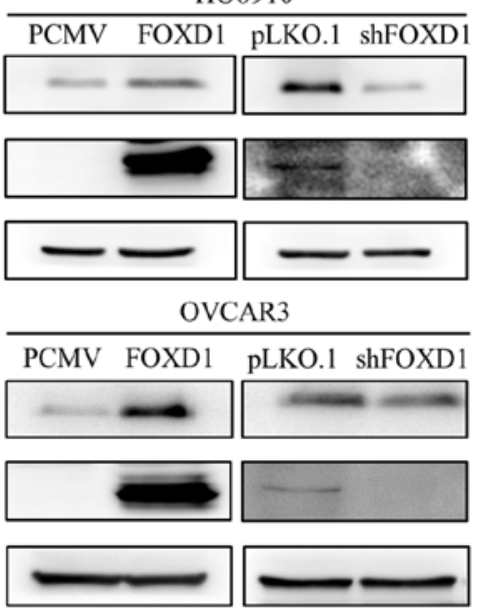

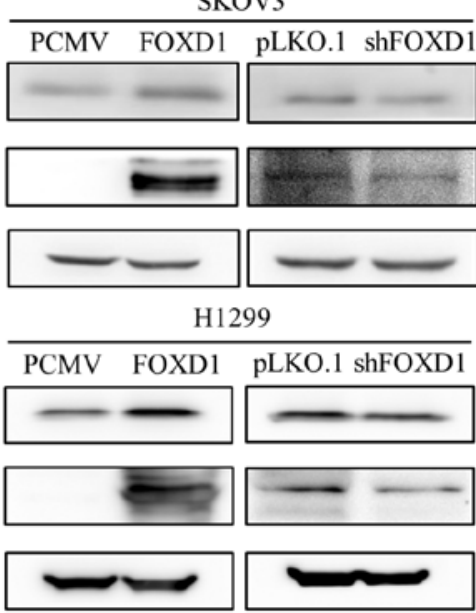

Figure 5. FOXD1 binds to the p21 promoter and induces its expression. (A) Three putative TFBSs of FOXD1 in the p21 promoter and the corresponding mutant sequence. (B) 293T cells were co-transfected with luciferase reporter vectors, each containing one of the TFBSs, and a FOXD1 overexpression vector or empty control vector. Luciferase activity was measured at $48 \mathrm{~h}$ following transfection. (C) 293T cells were co-transfected with luciferase reporters containing mutant TFBSs and a FOXD1 overexpression vector or empty vector control. Luciferase activity was determined at $48 \mathrm{~h}$ following transfection. (D) A2780 cells stably expressing FOXD1 were used to perform chromatin immunoprecipitation assay analysis. The immunoprecipitated protein-chromatin structures were then subjected to PCR analysis. (E) Western blot analysis demonstrated that manipulation of FOXD1 expression modulated p21 expression in six cancer cell lines. The results are expressed as the mean \pm standard deviation. ${ }^{* *} \mathrm{P}<0.01$ and ${ }^{* * * *} \mathrm{P}<0.001$, as indicated. FOXD1, forkhead box D1; TFBSs, transcription factor binding sites; PCMV, pLenti-C-Myc-DDK-IRES-Puro tagged vector; WT, wild-type; MUT, mutant; IgG, immunoglobulin G; shFOXD1, short hairpin RNA targeting FOXD1.

line following overexpression or silencing of FOXD1 expression was then evaluated. As demonstrated in Fig. 5E, FOXD1 overexpression was associated with an increase in $\mathrm{p} 21$ protein expression, while a reduction in FOXD1 was associated with a decrease in p21 expression. These results, together with those obtained from the immunohistochemical analysis of $\mathrm{p} 21$ expression in ovarian tumor xenografts, suggest that FOXD1 may bind to the promoter of p21 directly to regulate its expression in vitro and in vivo.

Knockdown of p 21 in FOXD1 overexpressing cells restores cell viability. To further investigate the role of FOXD1 in ovarian cancer cell proliferation via increasing p21 transcription, a rescue assay was performed by co-transfecting FOXD1 and p21-specific siRNA sequences in A2780 cells. The knockdown efficiency of the two p21-siRNAs is demonstrated in Fig. 6A. As indicated in Fig. 6B and C, the viability and clonogenicity of cells transfected with p21 siRNA and FOXD2 overexpression vectors was restored when compared with FOXD1 overexpressing cells transfected with siRNA controls. The results suggest that FOXD1 inhibits the proliferation of ovarian cancer cells via the direct targeting of p21.

\section{Discussion}

FOXD1 is known to function as an important transcription factor, which is involved in a number of biological processes (51). It has been demonstrated to function as an oncogene in lung, breast, brain and liver cancers (10-12). By contrast, microarray analyses have indicated that FOXD1 is downregulated in ovarian cancer patients (14). Consistently, the results of the current study demonstrated that FOXD1 
A

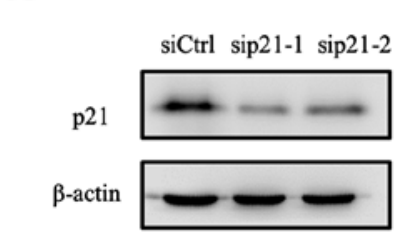

B

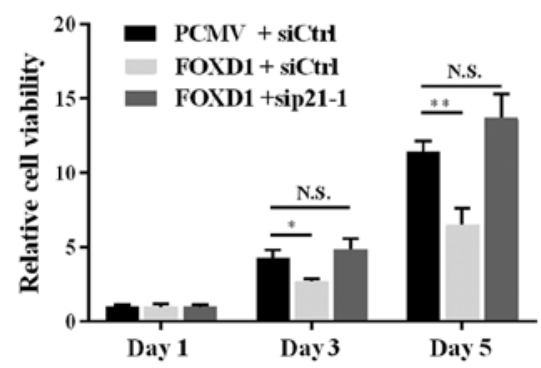

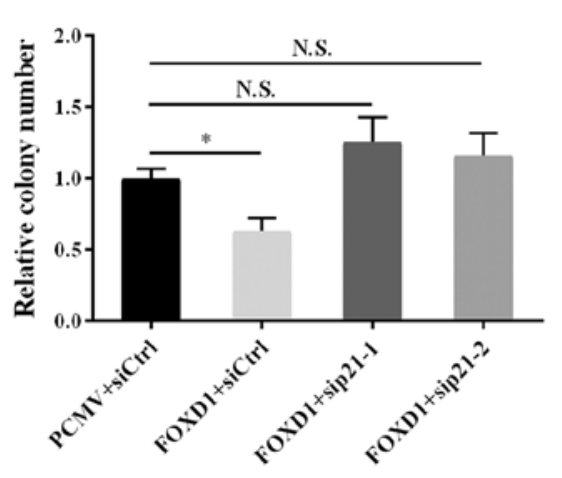

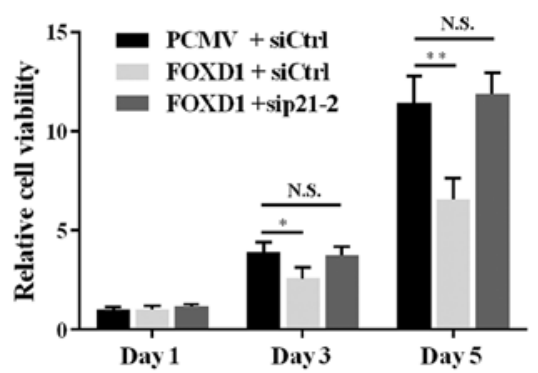

C PCMV+siCtrl FOXD1+sip21-1

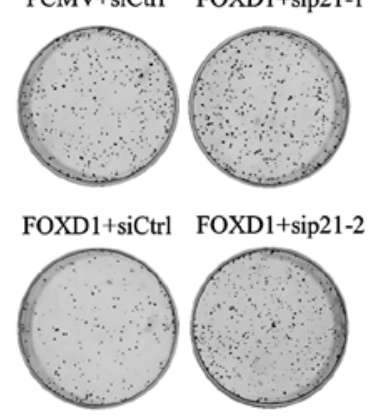

Figure 6. Knockdown of p21 restores cell viability in FOXD1-overexpressing cells. (A) Western blot analysis of p21 protein expression in A2780 transfected with two different p21-specific siRNAs or an siCtrl. (B) An MTT assay was used to determine the proliferation of A2780 cells co-transfected with FOXD1 and p21-specific siRNAs. (C) Following co-transfection with two different p21-siRNAs and an FOXD1 overexpression vector, the clonogenicity of A2780 cells was restored. The results are expressed as the mean \pm standard deviation. ${ }^{~} \mathrm{P}<0.05$ and ${ }^{* *} \mathrm{P}<0.01$, as indicated. FOXD1, forkhead box D1; siRNA, small interfering RNA; siCtrl, siRNA control; PCMV, pLenti-C-Myc-DDK-IRES-Puro tagged vector; N.S., not significant.

expression is decreased at the mRNA and protein levels in HGSOC patients. In addition, a previous study observed downregulation of FOXD1 in chemo-resistant ovarian cancer patients (52). Previous reports have also demonstrated that overexpression of $\mathrm{p} 21$ in SKOV3 and OVCAR3 cells lead to increased cisplatin sensitivity (53). The results of the present study indicated that FOXD1 directly promotes p21 expression, which may explain the association between FOXD1 and chemosensitivity in ovarian cancer. However further validation is required to establish this relationship.

miR-30a-5p serves an important role in cancer development and primarily influences the proliferation, metastasis, and autophagy of cancer cells (29). In the majority of cancer types, it functions as a tumor suppressor (29); however, in ovarian serous adenocarcinoma patients, miR-30a-5p was observed to be upregulated in urine samples, tissue samples and cell lines (30). Consistent with these observations, the results of the current study indicated that miR-30a-5p is elevated in HGSOC patient tissue samples. A previous report demonstrated that breast cancer cells co-transfected with miR-30a-5p and a luciferase reporter plasmid containing the 3'-UTR of FOXD1 mRNA, exhibited decreased luciferase activity (54). The current study identified the miR-30a-5p binding site in the 3'-UTR of FOXD1 mRNA. Previous studies have indicated that miR-30a-5p is upregulated in drug-resistant ovarian cancer cell lines $(31,32)$. As FOXD1 has been observed to be downregulated in chemoresistant ovarian cancer patients (52), the association between miR-30a-5p, FOXD1 and p21 may explain the drug resistance in ovarian cancer and provide a basis for further investigation. According to previous reports, miR-200a-5p is upregulated in HGSOC tissues (35). In addition, the expression of serum miR-200a-5p levels was significantly higher in patients with the serous subtype when compared with those presenting with clear cell, endometrioid or undifferentiated subtypes ovarian cancer subtypes (34). The results of the current study verified that miR-200a-5p is upregulated in HGSOC tissues. The elevated expression of these two miRNAs may explain why FOXD1 is downregulated in HGSOC.

p21 was the first p53-effector gene discovered; however, it is also known to be induced by p53-independent signaling pathways (16-19). The results of the present study demonstrated that FOXD1 inhibited the proliferation of five ovarian cancer cell lines and binds to the promoter of p21 in 293T cells. The p53 status of these five ovarian cancer cell lines are as follows: A2780 and HO8910, wild-type; OVCAR3 and CAOV3, mutant; SKOV3, controversial (null or mutant) (55-57). Therefore, FOXD1 induces p21 expression in ovarian cancer cells irrespective of the p53 status. To confirm that the inhibitory effects of FOXD1 overexpression on cancer cell proliferation is p53-independent and involves p21, the p53-null H1299 human lung cancer cell line was used. The results indicated that the inhibitory effect of FOXD1 on the growth of H1299 cells was similar to that of ovarian cancer cells, and that the regulation of p21 by FOXD1 does not involve p53. p21 is a multi-functional genome guardian depending on its subcellular localization (19). The nuclear accumulation of p21 induces cell cycle arrest and growth inhibition, while cytoplasmic accumulation promotes cell growth and survival $(58,59)$. Nuclear $\mathrm{p} 21$ therefore functions as a tumor suppressor. The results of the current study demonstrated that $\mathrm{p} 21$ expression was increased with forced FOXD1 expression, and that this interaction likely occurred in the nucleus. Therefore, these results suggest that the nuclear accumulation of p21 may function as a tumor suppressor in ovarian cancer. 
In conclusion, the results of the present study demonstrate that FOXD1 is downregulated in HGSOC, and the decreased level of FOXD1 predicts poor prognosis. In addition, FOXD1 was observed to inhibit the proliferation of ovarian cancer cells in vivo and in vitro, and was demonstrated to bind to the promoter of p21 directly. Furthermore, the expression of miR-30a-5p and miR-200a-5p was observed to be upregulated in HGSOC. These miRNA sequences were demonstrated to bind to the 3'-UTR of FOXD1 mRNA. Therefore, these results suggest that FOXD1 functions as a tumor suppressor in HGSOC and may serve as a biomarker or therapeutic target for HGSOC.

\section{Acknowledgements}

The authors would like to thank the staff at the Department of Pathology of Qilu Hospital (Shandong, China) for their technical support.

\section{Funding}

This study was supported by the National Natural Science Foundation of China (grant no. 81572554) and the National Clinical Research Center for Gynecological Oncology (grant no. 2015BAI13B05).

\section{Availability of data and materials}

All data generated or analyzed during this study are included in this published article.

\section{Author's contributions}

YW was a major contributor in designing the research, performing statistical analysis and writing the manuscript. CQ collected the tumor samples and interpreted data from immunohistochemistry analyses. NL and ZL provided technical support. CJ produced the lentiviruses and performed cell infection studies. CS performed experiments involving miRNA sequences. HB and SD preformed the chromatin immunoprecipitation experiments. HY performed the immunohistochemistry experiments. BK conceived and supervised the study. All authors have read and approved the final manuscript.

\section{Ethics approval and consent to participate}

Ethical approval was obtained from Ethics Committee of Shandong University Qilu Hospital and Ethics Committee on Animal Experiments of Shandong University Qilu Hospital (Shandong, China).

\section{Consent for publication}

Not applicable.

\section{Competing interests}

The authors declare that they have no competing interests.

\section{References}

1. Chen W, Zheng R, Baade PD, Zhang S, Zeng H, Bray F, Jemal A, Yu XQ and He J: Cancer statistics in China, 2015. CA Cancer J Clin 66: 115-132, 2016.

2. Reid BM, Permuth JB and Sellers TA: Epidemiology of ovarian cancer: A review. Cancer Biol Med 14: 9-32, 2017.

3. McCluggage WG: Morphological subtypes of ovarian carcinoma: A review with emphasis on new developments and pathogenesis. Pathology 43: 420-432, 2011.

4. Prat J: Ovarian carcinomas: Five distinct diseases with different origins, genetic alterations, and clinicopathological features. Virchows Arch 460: 237-249, 2012.

5. Bowtell DD, Böhm S, Ahmed AA, Aspuria PJ, Bast RC Jr, Beral V, Berek JS, Birrer MJ, Blagden S, Bookman MA, et al: Rethinking ovarian cancer II: Reducing mortality from highgrade serous ovarian cancer. Nat Rev Cancer 15: 668-679, 2015.

6. Carlsson P and Mahlapuu M: Forkhead transcription factors: Key players in development and metabolism. Dev Biol 250: 1-23, 2002.

7. Carreres MI, Escalante A, Murillo B, Chauvin G, Gaspar P, Vegar $\mathrm{C}$ and Herrera E: Transcription factor Foxd1 is required for the specification of the temporal retina in mammals. J Neurosci 31: 5673-5681, 2011.

8. Fetting JL, Guay JA, Karolak MJ, Iozzo RV, Adams DC, Maridas DE, Brown AC and Oxburgh L: FOXD1 promotes nephron progenitor differentiation by repressing decorin in the embryonic kidney. Development 141: 17-27, 2014.

9. Koga M, Matsuda M, Kawamura T, Sogo T, Shigeno A, Nishida E and Ebisuya M: Foxd1 is a mediator and indicator of the cell reprogramming process. Nat Commun 5: 3197, 2014.

10. Zhao YF, Zhao JY, Yue H, Hu KS, Shen H, Guo ZG and Su XJ: FOXD1 promotes breast cancer proliferation and chemotherapeutic drug resistance by targeting p27. Biochem Biophys Res Commun 456: 232-237, 2015.

11. Nakayama S, Soejima K, Yasuda H, Yoda S, Satomi R, Ikemura S Terai $\mathrm{H}$, Sato $\mathrm{T}$, Yamaguchi N, Hamamoto J, et al: FOXD1 expression is associated with poor prognosis in non-small cell lung cancer. Anticancer Res 35: 261-268, 2015.

12. Gao YF, Zhu T, Mao XY, Mao CX, Li L, Yin JY, Zhou HH and Liu ZQ: Silencing of Forkhead box D1 inhibits proliferation and migration in glioma cells. Oncol Rep 37: 1196-1202, 2017.

13. Chen J, Qian Z, Li F, Li J and Lu Y: Integrative analysis of microarray data to reveal regulation patterns in the pathogenesis of hepatocellular carcinoma. Gut Liver 11: 112-120, 2017.

14. Jiang X, Zhu T, Yang J, Li S, Ye S, Liao S, Meng L, Lu Y and Ma D: Identification of novel epithelial ovarian cancer biomarkers by cross-laboratory microarray analysis. J Huazhong Univ Sci Technolog Med Sci 30: 354-359, 2010.

15. Liu S, Bishop WR and Liu M: Differential effects of cell cycle regulatory protein $\mathrm{p} 21(\mathrm{WAF} 1 / \mathrm{Cip} 1)$ on apoptosis and sensitivity to cancer chemotherapy. Drug Resist Updat 6: 183-195, 2003.

16. el-Deiry WS, Tokino T, Velculescu VE, Levy DB, Parsons R, Trent JM, Lin D, Mercer WE, Kinzler KW and Vogelstein B: WAF1, a potential mediator of p53 tumor suppression. Cell 75: 817-825, 1993.

17. el-Deiry WS, Harper JW, O'Connor PM, Velculescu VE, Canman CE, Jackman J, Pietenpol JA, Burrell M, Hill DE, Wang Y, et al: WAF1/CIP1 is induced in p53-mediated G1 arrest and apoptosis. Cancer Res 54: 1169-1174, 1994.

18. Abbas T and Dutta A: p21 in cancer: Intricate networks and multiple activities. Nat Rev Cancer 9: 400-414, 2009.

19. Georgakilas AG, Martin OA and Bonner WM: p21: A two-faced genome guardian. Trends Mol Med 23: 310-319, 2017.

20. Shiohara M, el-Deiry WS, Wada M, Nakamaki T, Takeuchi S, Yang R, Chen DL, Vogelstein B and Koeffler HP: Absence of WAF1 mutations in a variety of human malignancies. Blood 84 : 3781-3784, 1994.

21. McKenzie KE, Siva A, Maier S, Runnebaum IB, Seshadri R and Sukumar S: Altered WAF1 genes do not play a role in abnormal cell cycle regulation in breast cancers lacking p53 mutations. Clin Cancer Res 3: 1669-1673, 1997.

22. Bukholm IK and Nesland JM: Protein expression of p53, p21 (WAF1/CIP1), bcl-2, Bax, cyclin D1 and pRb in human colon carcinomas. Virchows Arch 436: 224-228, 2000.

23. Caffo O, Doglioni C, Veronese S, Bonzanini M, Marchetti A, Buttitta F, Fina P, Leek R, Morelli L, Palma PD, et al: Prognostic value of p21(WAF1) and p53 expression in breast carcinoma: An immunohistochemical study in 261 patients with long-term follow-up. Clin Cancer Res 2: 1591-1599, 1996. 
24. Ogawa M, Onoda N, Maeda K, Kato Y, Nakata B, Kang SM, Sowa $\mathrm{M}$ and Hirakawa K: A combination analysis of p53 and p21 in gastric carcinoma as a strong indicator for prognosis. Int J Mol Med 7: 479-483, 2001.

25. Lu X, Toki T, Konishi I, Nikaido T and Fujii S: Expression of p21WAF1/CIP1 in adenocarcinoma of the uterine cervix: A possible immunohistochemical marker of a favorable prognosis. Cancer 82: 2409-2417, 1998.

26. Bartel DP: MicroRNAs: Target recognition and regulatory functions. Cell 136: 215-233, 2009.

27. Yang SJ, Yang SY, Wang DD, Chen X, Shen HY, Zhang XH, Zhong SL, Tang JH and Zhao JH: The miR-30 family: Versatile players in breast cancer. Tumour Biol 39: 1010428317692204, 2017.

28. Feng X, Wang Z, Fillmore R and Xi Y: MiR-200, a new star miRNA in human cancer. Cancer Lett 344: 166-173, 2014.

29. Yang X, Chen Y and Chen L: The versatile role of microRNA-30a in human cancer. Cell Physiol Biochem 41: 1616-1632, 2017.

30. Zhou J, Gong G, Tan H, Dai F, Zhu X, Chen Y, Wang J, Liu Y Chen $\mathrm{P}, \mathrm{Wu} \mathrm{X}$, et al: Urinary microRNA-30a-5p is a potential biomarker for ovarian serous adenocarcinoma. Oncol Rep 33 2915-2923, 2015

31. Chen N, Chon HS, Xiong Y, Marchion DC, Judson PL, Hakam A, Gonzalez-Bosquet J, Permuth-Wey J, Wenham RM, Apte SM, et al: Human cancer cell line microRNAs associated with in vitro sensitivity to paclitaxel. Oncol Rep 31: 376-383, 2014.

32. Liu J, Wu X, Liu H, Liang Y, Gao X, Cai Z, Wang W and Zhang H: Expression of microRNA-30a-5p in drug-resistant and drug-sensitive ovarian cancer cell lines. Oncol Lett 12 : 2065-2070, 2016.

33. Wyman SK, Parkin RK, Mitchell PS, Fritz BR, O'Briant K, Godwin AK, Urban N, Drescher CW, Knudsen BS and Tewari M: Repertoire of microRNAs in epithelial ovarian cancer as determined by next generation sequencing of small RNA cDNA libraries. PLoS One 4: e5311, 2009.

34. Zuberi M, Mir R, Das J, Ahmad I, Javid J, Yadav P, Masroor M, Ahmad S, Ray PC and Saxena A: Expression of serum miR-200a, miR-200b, and miR-200c as candidate biomarkers in epithelial ovarian cancer and their association with clinicopathological features. Clin Transl Oncol 17: 779-787, 2015.

35. Vilming Elgaaen B, Olstad OK, Haug KB, Brusletto B Sandvik L, Staff AC, Gautvik KM and Davidson B: Global miRNA expression analysis of serous and clear cell ovarian carcinomas identifies differentially expressed miRNAs including miR-200c-3p as a prognostic marker. BMC Cancer 14: 80, 2014.

36. Katz B, Tropé CG, Reich R and Davidson B: MicroRNAs in ovarian cancer. Hum Pathol 46: 1245-1256, 2015.

37. Livak KJ and Schmittgen TD: Analysis of relative gene expression data using real-time quantitative PCR and the 2(-Delta Delta C(T)) method. Methods 25: 402-408, 2001

38. Wu X, Yang N, Zhou WH, Xu J, Chen JJ, Zheng FM, Long ZJ, Yue CF, Ai KX, Liu LL, et al: Up-regulation of p21 inhibits TRAIL-mediated extrinsic apoptosis, contributing resistance to SAHA in acute myeloid leukemia cells. Cell Physiol Biochem 34: 506-518, 2014

39. Rao A, Coan A, Welsh JE, Barclay WW, Koumenis C and Cramer SD: Vitamin D receptor and p21/WAF1 are targets of genistein and 1,25-dihydroxyvitamin D3 in human prostate cancer cells. Cancer Res 64: 2143-2147, 2004.

40. Kobayashi A, Mugford JW, Krautzberger AM, Naiman N, Liao J and McMahon AP: Identification of a multipotent self-renewing stromal progenitor population during mammalian kidney organogenesis. Stem Cell Reports 3: 650-662, 2014.

41. Filtz TM, Vogel WK and Leid M: Regulation of transcription factor activity by interconnected post-translational modifications. Trends Pharmacol Sci 35: 76-85, 2014.

42. Gan L, Zheng W, Chabot JG, Unterman TG and Quirion R: Nuclear/cytoplasmic shuttling of the transcription factor FoxO1 is regulated by neurotrophic factors. J Neurochem 93: 1209-1219, 2005 .
43. Harper JV: Synchronization of cell populations in G1/S and G2/M phases of the cell cycle. Methods Mol Biol 296: 157-166, 2005.

44. Tothill RW, Tinker AV, George J, Brown R, Fox SB, Lade S, Johnson DS, Trivett MK, Etemadmoghadam D, Locandro B, et al: Novel molecular subtypes of serous and endometrioid ovarian cancer linked to clinical outcome. Clin Cancer Res 14: 5198-5208, 2008.

45. Camp RL, Dolled-Filhart M and Rimm DL: X-tile: A new bio-informatics tool for biomarker assessment and outcomebased cut-point optimization. Clin Cancer Res 10: 7252-7259, 2004.

46. Agarwal V, Bell GW, Nam JW and Bartel DP: Predicting effective microRNA target sites in mammalian mRNAs. eLife 4: $4,2015$.

47. Betel D, Wilson M, Gabow A, Marks DS and Sander C: The microRNA.org resource: Targets and expression. Nucleic Acids Res 36: D149-D153, 2008

48. Cartharius K, Frech K, Grote K, Klocke B, Haltmeier M, Klingenhoff $A$, Frisch $M$, Bayerlein $M$ and Werner $T$ : MatInspector and beyond: Promoter analysis based on transcription factor binding sites. Bioinformatics 21: 2933-2942, 2005.

49. Mathelier A, Fornes O, Arenillas DJ, Chen CY, Denay G, Lee J, Shi W, Shyr C, Tan G, Worsley-Hunt R, et al: JASPAR 2016: A major expansion and update of the open-access database of transcription factor binding profiles. Nucleic Acids Res 44: D110-D115, 2016.

50. Bell D, Berchuck A, Birrer M, Chien J, Cramer DW, Dao F, Dhir R, DiSaia P, Gabra H, Glenn P, et al; Cancer Genome Atlas Research Network: Integrated genomic analyses of ovarian carcinoma. Nature 474: 609-615, 2011.

51. Pierrou S, Hellqvist $M$, Samuelsson L, Enerbäck $S$ and Carlsson P: Cloning and characterization of seven human forkhead proteins: Binding site specificity and DNA bending. EMBO J 13: 5002-5012, 1994.

52. Ju W, Yoo BC, Kim IJ, Kim JW, Kim SC and Lee HP: Identification of genes with differential expression in chemoresistant epithelial ovarian cancer using high-density oligonucleotide microarrays. Oncol Res 18: 47-56, 2009.

53. Lincet H, Poulain L, Remy JS, Deslandes E, Duigou F, Gauduchon P and Staedel C: The p21(cip1/waf1) cyclin-dependent kinase inhibitor enhances the cytotoxic effect of cisplatin in human ovarian carcinoma cells. Cancer Lett 161: 17-26, 2000.

54. Ouzounova M, Vuong T, Ancey PB, Ferrand M, Durand G, Le-Calvez Kelm F, Croce C, Matar C, Herceg Z and HernandezVargas H: MicroRNA miR-30 family regulates non-attachment growth of breast cancer cells. BMC Genomics 14: 139, 2013.

55. Forbes S, Clements J, Dawson E, Bamford S, Webb T, Dogan A, Flanagan A, Teague J, Wooster R, Futreal PA, et al: COSMIC 2005. Br J Cancer 94: 318-322, 2006.

56. Soussi T: Handbook of p53 Mutation in Cell Lines. Version 1, 2007.

57. Su WJ, Fang JS, Cheng F, Liu C, Zhou F and Zhang J: RNF2/ Ring $1 b$ negatively regulates $\mathrm{p} 53$ expression in selective cancer cell types to promote tumor development. Proc Natl Acad Sci USA 110: 1720-1725, 2013.

58. Piccolo MT and Crispi S: The dual role played by p21 may influence the apoptotic or anti-apoptotic fate in cancer. J Cancer Res Updates 1: 189-202, 2012.

59. Gawriluk TR, Simkin J, Thompson KL, Biswas SK, ClareSalzler Z, Kimani JM, Kiama SG, Smith JJ, Ezenwa VO and Seifert AW: Comparative analysis of ear-hole closure identifies epimorphic regeneration as a discrete trait in mammals. Nat Commun 7: 11164, 2016. 